\title{
THE LYNDON-HOCHSCHILD-SERRE SPECTRAL SEQUENCES FOR SHEAVES WITH OPERATORS
}

\author{
TATSUO SUWA ${ }^{1}$
}

\begin{abstract}
Two spectral sequences associated with a $G$-sheaf and a normal
\end{abstract} subgroup of $G$ are given together with an application.

If $H$ is a normal subgroup of a group $G$, the Lyndon-Hochschild-Serre spectral sequence relates the cohomology of $H$ and of $G / H$ to that of $G$. In this note we give two analogous spectral sequences in the cohomology theory of groups with coefficients in sheaves with operators. Applications include a direct proof of a theorem of Conner and Raymond [1].

1. Sheaves with operators. We start by briefly reviewing the cohomology of groups with coefficients in sheaves with operators. For details, we refer to [1], [2], and [3]. Let $(G, X)$ be a continuous action of a group $G$ on a topological space $X$. A $G$-sheaf over $X$ is a sheaf $\delta$ of abelian groups over $X$ with an action $(G, \delta)$ such that the projection $\delta \rightarrow X$ is equivariant and that for each $g \in G$ and $x \in X, g: \delta_{x} \rightarrow \delta_{g x}$ is a group homomorphism between the stalks. The categories of $G$-sheaves over $X$ and of abelian groups are denoted, respectively, by $\mathcal{C}_{X}^{G}$ and $\mathcal{C}$. The functor $\Gamma_{X}^{G}: \mathcal{C}_{X}^{G} \rightarrow \mathcal{C}$ sends a $G$-sheaf $\mathcal{S}$ to the group $\Gamma(X, \delta)^{G}$ of $G$-invariant sections of $\delta$ over $X$. For a $G$-sheaf $\delta$ over $X$, the cohomology $H \cdot(G, \delta)$ of $G$ with coefficients in $\delta$ is defined by $H \cdot(G, \delta)$ $=R \cdot \Gamma_{X}^{G}(\mathcal{S})$, where $R \cdot \Gamma_{X}^{G}$ denotes the right derived functor of $\Gamma_{X}^{G}$.

2. The Lyndon-Hochschild-Serre spectral sequences for $G$-sheaves. Let $H$ be a normal subgroup of $G$ and let $\varphi: X \rightarrow X / H$ be the canonical projection onto the orbit space. The category $\bigodot_{X}^{G}$ can be thought of as a subcategory of $\mathcal{C}_{X}^{H}$ in a natural manner. If $M$ is a $G$-module, then the group $\Gamma^{H}(M)=M^{H}$ of $H$-invariant elements of $H$ becomes naturally a $G / H$-module. Thus the functor $\Gamma_{X}^{H}: \bigodot_{X}^{H} \rightarrow \bigodot$ sends $\bigodot_{X}^{G}$ into the category $\bigodot^{G / H}$ of $G / H$-modules, since it is factorized as $\Gamma_{X}^{H}=\Gamma^{H} \circ \Gamma_{X}$. We denote by $\mathcal{C}_{X / H}$ the category of sheaves of abelian groups over $X / H$. The functor $\varphi_{*}^{H}: \mathcal{C}_{X}^{H} \rightarrow \mathcal{C}_{X / H}$ assigns, by definition, to each $H$-sheaf $\mathcal{S}$ the sheaf over $X / H$ determined by the presheaf $U \rightarrow \Gamma\left(\varphi^{-1}(U), \delta\right)^{H}$. The restriction of $\varphi_{*}^{H}$ to the subcategory $\mathcal{C}_{X}^{G}$

Received by the editors December 2, 1978.

AMS (MOS) subject classifications (1970). Primary 18G40, 18H10, 55B30; Secondary 32C35.

Key words and phrases. Lyndon-Hochschild-Serre spectral sequence, cohomology of groups with coefficients in sheaves with operators.

${ }^{1}$ Partially supported by the National Science Foundation and the Sakkokai Foundation. 
can be thought of as a functor into the category $\sum_{X / H}^{G / H}$ of $G / H$-sheaves over $X / H$ in a natural manner.

LEMMA. If $\mathcal{G}$ is an injective $G$-sheaf over $X$, then

(1) $g$ is an injective $H$-sheaf over $X$,

(2) $\Gamma_{X}^{H}(9)$ is an injective $G / H$-module,

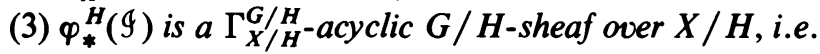

$$
R^{n} \Gamma_{X / H}^{G / H}\left(\varphi_{*}^{H}(\mathcal{G})\right)\left(=H^{n}\left(G / H, \varphi_{*}^{H}(\mathcal{G})\right)\right)=0, \text { for } n>1 \text {. }
$$

Proof. (1) Set $O=\mathbf{Z}(H)$ in [2, Lemma 5.6.2]. (2) Recall the factorization $\Gamma_{X}^{H}=\Gamma^{H} \circ \Gamma_{X}$. By [2, Corollaire de Proposition 5.1.3], $\Gamma_{X}$ sends an injective $G$-sheaf to an injective $G$-module. On the other hand, $\Gamma^{H}$ sends an injective $G$-module to an injective $G / H$-module. (3) By (1) above and [2, Corollaire de Proposition 5.1.3], the sheaf $\varphi_{*}^{H}(\mathcal{G})$ is a flabby sheaf over $X / H$. Consider the

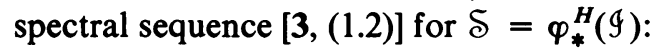

$$
E_{2}^{p, q}=H^{p}\left(G / H, H^{q}\left(X / H, \varphi_{*}^{H}(\mathcal{G})\right)\right) \Rightarrow H^{n}\left(G / H, \varphi_{*}^{H}(g)\right) .
$$

Since $\varphi_{*}^{H}(9)$ is flabby, the spectral sequence degenerates to yield

$$
\begin{aligned}
H^{n}\left(G / H, \varphi_{*}^{H}(\Im)\right) & \simeq H^{n}\left(G / H, H^{0}\left(X / H, \varphi_{*}^{H}(\Im)\right)\right) \\
& =H^{n}\left(G / H, H^{0}(X, \Im)^{H}\right) .
\end{aligned}
$$

Also consider the Lyndon-Hochschild-Serre spectral sequence for the $G$-module $H^{0}(X, 9)$ :

$$
E_{2}^{p, q}=H^{p}\left(G / H, H^{q}\left(H, H^{0}(X, 9)\right)\right) \Rightarrow H^{n}\left(G, H^{0}(X, 9)\right) .
$$

By (1) above and [2, Corollaire de Proposition 5.1.3], $H^{0}(X, \mathscr{G})=\Gamma_{X}(\mathscr{G})$ is injective either as $G$-module or as $H$-module. Hence $H^{n}\left(H, H^{0}(X, 9)\right)=0=$ $H^{n}\left(G, H^{0}(X, 9)\right)$ for $n \geqslant 1$. Therefore we have $H^{n}\left(G / H, H^{0}(X, g)^{H}\right)=$ $H^{n}\left(G / H, H^{0}\left(H, H^{0}(X, \mathscr{G})\right)\right)=H^{n}\left(G, H^{0}(X, 9)\right)=0$, for $n>1$. Q.E.D.

THEOREM. If $\mathcal{S}$ is a $G$-sheaf over $X$ and if $H$ is a normal subgroup of $G$, then for each nonnegative integer $q$, the group $H^{q}(H, \delta)$ has a canonical structure of $G / H$-module and the sheaf $R^{q} \varphi_{*}^{H}(\mathcal{S})$ that of $G / H$-sheaf over $X / H$ and there are two spectral sequences

$$
\begin{aligned}
& { }^{\prime} E_{2}^{p, q}=H^{p}\left(G / H, R^{q} \varphi_{*}^{H}(\delta)\right) \Rightarrow H^{n}(G, \delta), \\
& { }^{\prime \prime} E_{2}^{p, q}=H^{p}\left(G / H, H^{q}(H, \delta)\right) \Rightarrow H^{n}(G, \delta) .
\end{aligned}
$$

Proof. Lemma (1) shows that $R \cdot \Gamma_{X}^{H}(\delta)$ and $R \cdot \varphi_{*}^{H}(\delta)$ can be computed by taking a $G$-injective resolution of $\delta$. Thus, for each $q, H^{q}(H, \delta)=R^{q} \Gamma_{X}^{H}(\delta)$ has a canonical structure of $G / H$-module and $R^{q} \varphi_{*}^{H}(\delta)$ that of $G / H$-sheaf over $X / H$. With the aid of the lemma, [2, Théorème 2.4.1] is applied to the 
commutative diagram

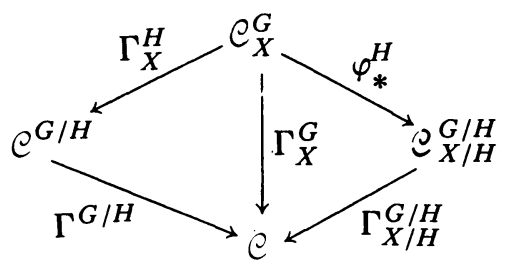

to obtain the spectral sequences.

3. An application. (3.10) Theorem in [1] is obtained from the above theorem as follows. Let $(N, W)$ be a properly discontinuous action of a group $N$ on $W$. A representation $\Phi: N \rightarrow \mathrm{GL}(2 k, \mathrm{Z})$ defines an $N$-sheaf structure on the constant sheaf $\mathscr{Z}^{2 k}=W \times \mathbf{Z}^{2 k}$. Let $L \subset N$ be a normal subgroup satisfying

(i) $L$ acts freely on $W$,

(ii) $L \subset \operatorname{Ker} \Phi$.

Denote by $\mu$ : $W \rightarrow W / L=B$ the canonical projection onto the orbit space. By (ii), $\Phi$ defines an $N / L$-sheaf structure on the constant sheaf $\mathscr{Z}^{2 k}=B \times$ $\mathbf{Z}^{2 k}$ on $B$. We set $X=W, G=N, H=L, \varphi=\mu$ and $\delta=\mathscr{Z}^{2 k}$ in the first spectral sequence of the theorem. The condition (i) implies that $R^{q} \mu_{*}^{L}\left(\mathscr{Z}^{2 k}\right)=$ 0 , for $q>1$. Hence the spectral sequence degenerates to yield $H^{n}\left(N / L, \mu_{*}^{L}\left(\mathscr{Z}^{2 k}\right)\right) \simeq H^{n}\left(N, \mathscr{Z}^{2 k}\right)$. Moreover, from conditions (i) and (ii), we see that the sheaf $\mu_{*}^{L}\left(\mathscr{Z}^{2 k}\right) \rightarrow B$ is identical with $\mathscr{Z}^{2 k} \rightarrow B$. Hence we get

$$
H^{n}\left(N / L, \mathscr{Z}^{2 k}\right) \simeq H^{n}\left(N, \mathscr{Z}^{2 k}\right) .
$$

For another application, see the proof of [3, Corollary 4.3].

\section{BIBLIOGRAPHY}

1. P. E. Conner and F. Raymond, Holomorphic Seifert fiberings, Proceedings of the Second Conference on Compact Transformation Groups, Part II, Lecture Notes in Math., vol. 299, Springer-Verlag, Berlin and New York, 1972, pp. 124-204.

2. A. Grothendieck, Sur quelques points d'algèbre homologique, Tôhoku Math. J. 9 (1957), 119-227.

3. T. Suwa, Deformations of holomorphic Seifert fiber spaces, Invent. Math. 51 (1979), 77-102.

Department of Mathematics, Hokkaido University, Sapporo, Japan 